\title{
Optical Sensor to Monitor Pupillary Light Reflex
}

\author{
K. RÓŻANOWsKI ${ }^{a, *}$ AND K. MURAWSKI ${ }^{b}$ \\ ${ }^{a}$ Military Institute of Aviation Medicine, Aviation Bioengineering Department \\ Z. Krasińskiego 54, 01-755 Warsaw, Poland \\ ${ }^{b}$ Military University of Technology, Institute of Teleinformatics and Automatics \\ S. Kaliskiego 2, 00-908 Warsaw, Poland
}

\begin{abstract}
The aim of the paper is to describe design of an optical sensor intended to analyze pupillary light reflex. It also presents the results of physiological adaptation mechanisms in human eye, i.e. response of the iris to changes in the intensity of light that falls on the retina of the eye under conditions of sensory deprivation. Pupillary light reflex is a closed loop nerve reflex. It controls the amount of light that reaches the retina. Based on the test results, an optical sensor was designed, fabricated and correctly calibrated. In comparative tests with the use of $\mathrm{F}^{2} \mathrm{D}$ Fit-For-Duty, a commercial system by AMTech, selected pupillographic parameters were primarily evaluated (baseline pupil diameter, oscillations, reflex latency, maximum reaction time, pupil constriction time, pupil dilation time, and constriction amplitude) under conditions of diminished alertness, reduced ability to concentrate, increasing fatigue, and drowsiness. The solution comes as part of a mobile pupillography device intended to be assembled in cars and airplanes to identify conditions of lower alertness, reduced ability to concentrate, increasing fatigue and drowsiness in drivers, pilots and traffic controllers, and to trigger alarm and preventive measures, if necessary.
\end{abstract}

DOI: $10.12693 /$ APhysPolA.124.558

PACS: 42.66.Ct, 42.30.Va, 07.05.Pj

\section{Introduction}

Described sensor is an interesting combination of hardware and software solutions. This class of optical sensors, using vision-based image analysis, is used in pupillometry and enables identification of eyeball movement, parameters describing the dynamics of the eyeball covering and dynamic pupil change reaction. The evaluation of pupil motion provides functional information about the autonomous nervous system [1]. Pupillary light reflex (PLR) is the iris's response to changes in the intensity of light incidence on the retina. This reflex is modulated by the accommodation state of the eye, and factors of sensory and emotional origins. Effectors are contractor and dilator muscles of the iris that try to maintain a constant level of retina illumination by changing the size of the pupil. Time characteristic of the pupillary reflex depends on the state of the two antagonistically acting systems of the autonomous nervous system (ANS): sympathetic nervous system (SNS) and parasympathetic nervous system (PNS).

Pupillometry was initially used in ophthalmology [1]. The pupillary size is significant for the interpretation of perimetry of the visual field and electroretinography and for the preoperative assessment of a refractive surgery [2]. It is also used in neuro ophthalmology for the description of lesion of the afferent or efferent nervous ways [3]. The PLR has already been studied to detect a PNS defect [4] in various affections: alcoholism, diabetes, AIDS, depression, anxiety, drug addiction, schizophrenia. Pupillogra-

*corresponding author; e-mail: krozan@wiml.waw.pl phy is also used in the study of Alzheimer's and Parkinson's diseases [5]. Because pupil dilation is regulated autonomically, this method was also tried as a valid measure of pain [6]. There is also research underway with using pupillometry to assess alertness in hypersomnolent patients. The phenomenon of the measurement behavior of the pupil is also used in clinically sleepy patients [7], and for the assessment of cardiac autonomic function in dynamic athletes [8]. This method has found a number of clinical applications. Identification of dynamic pupillary changes is also the subject of research on human behavior in various activity areas $[9,10]$. This is mainly due to the fact that it is possible to evaluate human psychophysiological state, which is influenced by the level of sleepiness or alertness by the so-called fatigue wave, which is the basis for the pupillographic sleepiness test (PST) [11]. An additional parameter referring to the pupil's tendency to instability is the pupillary unrest index (PUI). It is defined by cumulative changes in pupil size based on mean values of consecutive data sequences [12].

In the available literature, there is no comparative analysis of the indicators of the PST test, such as PUI, against the parameters of the pupillary reflex, as well as no interpretation of these indicators in relation to sensory and mental fatigue. The research took into account the indicators determined in the commercially available $\mathrm{F}^{2} \mathrm{D}$ Fit-For-Duty device by AMTech, which was used as the reference device. In the experiments, the pupillary reflex was researched, which is characterized by latency (L) which describes the delay between a light pulse and the pupil reaction, duration of reaction (DR), which is the time it takes to reach the minimum diameter, time of minimum diameter (TMD), which is the time for which the diameter is less than $50 \%$ of the beginning value, $2 / 3$ 
contraction interval $(2 / 3 \mathrm{CI})$, which is the time for which the amplitude reaches $1 / 3$ of the contraction phase value, $1 / 3$ redilatation interval $(1 / 3 \mathrm{RI})$, which represents the time for which the amplitude reaches $1 / 3$ of the redilatation phase value, and finally amplitude (A), which represents the difference between the maximum and minimum values of the diameter.

\section{Description of the experimental model and the test stand}

Design of the sensor for measuring PLR and PST as well parameters of the eyelid covering the eyeball were preceded by laboratory tests. The purpose of the research was to verify the possibility of identification of time-varying PLR behavior in relation to the PUI factor described in the literature. At the same time, the requirements for the designed optical sensor were collected. The resolution of the converter, frequency of image acquisition and required speed of the pupil detection algorithm were taken into account.

The study was conducted in two stages. The first step was research where assumptions were verified. The second stage consisted of a study of the designed PLR sensor. The first phase of the study was preceded with a series of experimental research of the commercially available $\mathrm{F}^{2} \mathrm{D}$ Fit-For-Duty device by AMTech. The experimental model assumed verification of PLR parameter usability in correlation with results of the pupillography sleepiness test PST. Research was performed on a group of 10 volunteers in a laboratory environment during at night. The average age of participants was $34.8 \pm 6.5$ years. Each participant was familiarized with the description of the experiment and agreed to participate in the study. The PLR measurements were taken on both eyeballs, which were exposed to a series of five flashes with the interval of $30 \mathrm{~s}$. The second phase of the experiment involved testing the constructed PLR sensor. Functional tests were also carried out on a group of 10 volunteers. The study took place in an ophthalmology darkroom, which provided necessary conditions for testing. Measurements were performed in the identical measurement workflow, as in the case of pilot studies [13].

\section{Test results}

Initial results showed high usability of the dedicated parameters characteristic for the PLR in estimating the level of fatigue and lowered concentration, as well as high correlation with the PUI index (Table I). Statistical analysis was performed using conventional statistical methods based on variance analysis. They were multi-dimensional, multi-factor analyses of identifiable variables from the pupillary reflex signal, categorized by Person, Task, Eye variables and the light excitation sequence (Profile). In the subsequent measurement times, parameters were estimated that described the relative amplitude and contraction velocity of the pupillary reflex (Table II).
Measurements for each person were made four times with the average interval of $2 \mathrm{~h}$ between hours 11.00 PM and 6.30 AM. These changes proved to be statistically significant.

Investigations shown that the difference between the first and last measurement point is $3.07 \%$. With the average value of the observed changes in pupil width of $1.77 \mathrm{~mm}$, the required measurement accuracy could not be less than $0.09 \mathrm{~mm}$. This value is necessary to design the sensor in order to observe the changes in the work of the ANS for the experimental model adopted. It was necessary to analyze the behavior of the contraction velocity parameter in order to determine the required speed of the optical system. The scope of averaged parameter values for the first and last measurement was $0.5 \mathrm{~mm} / \mathrm{s}$. It means that the measurement resolution cannot be less than $0.25 \mathrm{~mm} / \mathrm{s}$. These assumptions were adopted in the design of the PLR optical sensor.

TABLE I

Table showing the values of the rPUI and $\mathrm{rPUI}_{\text {rel }}$ parameters in successive measurement points.

\begin{tabular}{c|c|c|c|c|c}
\hline \hline & & \multicolumn{2}{|c|}{ rPUI } & \multicolumn{2}{c}{ rPUI $_{\text {rel }}$} \\
\hline task & $N$ & mean & st.d. & mean & st.d. \\
\hline All & 398 & 0.83 & 0.42 & 0.20 & 0.29 \\
0 & 99 & 0.62 & 0.25 & 0.00 & 0.00 \\
1 & 102 & 0.76 & 0.35 & 0.13 & 0.15 \\
2 & 98 & 0.91 & 0.40 & 0.28 & 0.17 \\
3 & 99 & 1.02 & 0.55 & 0.39 & 0.45
\end{tabular}

TABLE II

Table showing the values of the relative amplitude and contraction velocity indicators in successive measurement points.

\begin{tabular}{c|c|c|c|c|c}
\hline \hline & & \multicolumn{2}{|c|}{ Rel. amplitude } & \multicolumn{2}{c}{ Contr. velocity } \\
\hline Task & $N$ & mean & st.d. & mean & st.d. \\
\hline 0 & 79 & 24.21 & 5.11 & 3.98 & 0.82 \\
1 & 76 & 25.53 & 5.28 & 4.21 & 0.89 \\
2 & 80 & 26.22 & 5.36 & 4.29 & 0.83 \\
3 & 79 & 27.28 & 5.47 & 4.49 & 0.87
\end{tabular}

\section{Optical sensor to monitor pupillary light reflex}

In pupillary light reflex testing, it is most important to determine the parameters of pupil response to light stimulation with a specific duration $(t=250 \mathrm{~ms})$. The measurement method for PLR determines the design of the illuminator control system, image acquisition system and software. In the adopted implementation the change of pupil size in time is determined by analyzing the motion camera image. The acquired image is recorded in the near-infrared band $(\lambda=850 \mathrm{~nm})$ by the Optitrack V120 camera. The red KPTD-3216SURC LED with the wavelength of $\lambda=640 \mathrm{~nm}$ was used as an excitation source. 
Near-infrared light was used because of the lack of effect on the pupil. The choice of red light as the excitation was intuitive. There is no clear information in the literature that would help determine the color of the LED generating the light pulse (excitation). This research is still underway. The control system was separated from the illuminator plate in the device. The adopted solution, by changing the illuminator plate, enables to examine the behavior of the pupil at different light wavelengths. The control system was designed as a microcomputer system controlled by the RS-485 bus. The RS- 485 bus is controlled by the AT89s8253 microcontroller. It receives and executes commands issued from the host system (IBM PC type computer). These commands are encoded by the system and transmitted through the $\mathrm{I} 2 \mathrm{C}$ bus to the peripheral device. The PCA 9622DR LED control circuit made by NXP Semiconductor was used as the peripheral device. This circuit is a specialized controller of sixteen channels of light-emitting diodes, LED0-LED15. It enables selective brightness control for each channel. Brightness control is achieved by choosing the parameters of pulse width generators (PWM0-PWM15). An external resistor in series with the LED circuit sets the maximum current in the channel. This current should not exceed $100 \mathrm{~mA}$ at an input voltage of up to $40 \mathrm{~V}$.

The application used fourteen of the sixteen available channels. Each of them has a branch of LED supplied with a voltage of $U_{Z}=+5 \mathrm{~V}$.

Twelve HIRB5-43G-A infrared LEDs and two KPTD-3216SURC LEDs operating in the visible spectrum were placed on a separate printed circuit board. It is connected to the illuminator plate with the FFC elastic tape from Molex (MX-98266-0193). Visible light LEDs are used for excitation and communicating with the tested person. LEDs operated in the infrared spectrum, as the illuminator with almost any configurable layout and intensity of light. The resulting sensor was tested in a system with and without the infrared mirror. Experiments were carried out in a darkroom due to the need to isolate the pupil from the outside light. Ultimately, after the construction of the head-mounted housing, the sensor will operate in the infrared mirror configuration. Such a configuration will provide for greater flexibility and repeatability of tests, Figs. 1 and 2.

Software was developed for the constructed design to automatically search the pupil in the image, Fig. 3. The pupil searching algorithm uses techniques described in Refs. [14-16]. After finding the pupil, its size (width and height) is measured. Finally, because of the lower sensitivity to interference due to eyelid movements, the value equal to the width of the pupil is presented as a result of the measurement. The software measures the pupil size with the interval of $t=40 \mathrm{~ms}$ ( $25 \mathrm{fps})$. Without modifying the image processing algorithms, this value can be reduced to $t=10 \mathrm{~ms}$. As a result, measurement of changes in pupil size will be performed at a frequency of one hundred frames per second (100 fps). The time $t=40 \mathrm{~ms}$ was used because of the parameters of the reference de-

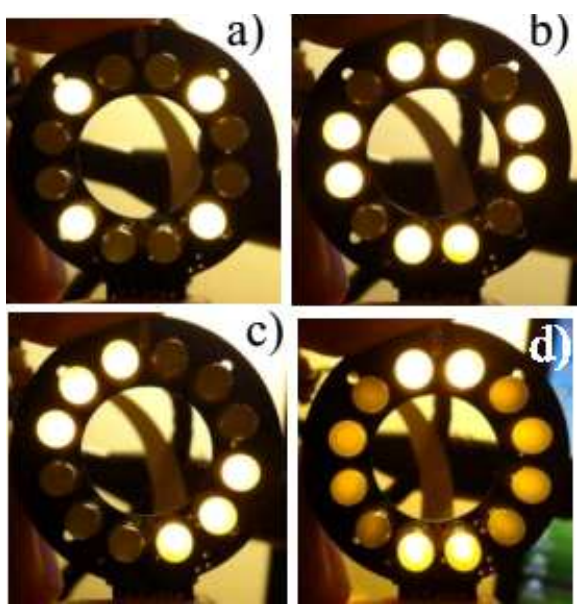

Fig. 1. The images of selected illuminator layouts: change in the layout of active LEDs (a)-(c), selective power control for each channel (d).
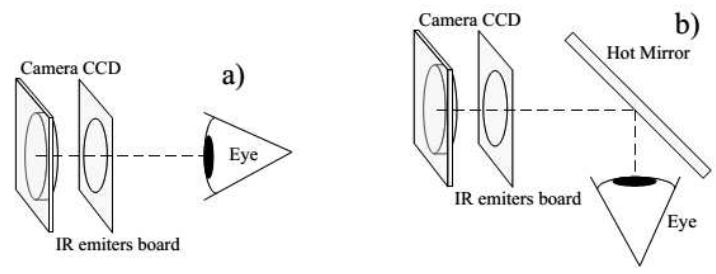

Fig. 2. Sensor operating layouts: without a mirror (a), with the infrared mirror (b).

vice $\mathrm{F}^{2} \mathrm{D}$ Fit-For-Duty by AMTech, which analyzes the image at the rate of $25 \mathrm{fps}$.

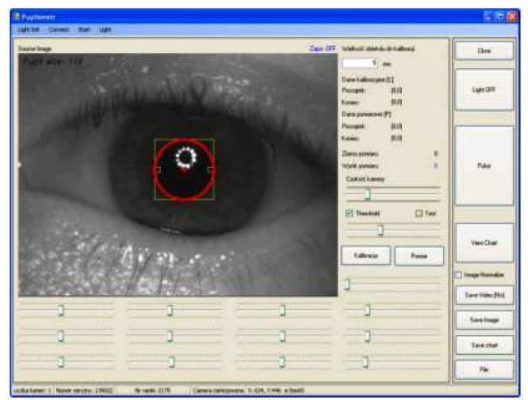

Fig. 3. View of the application interoperating with the PLR testing sensor.

The PLR measurement is performed by pressing the "Pulse" button. The software automatically generates a light pulse with a duration of $t=250 \mathrm{~ms}$, and then identifies and saves the changes in pupil size over $2 \mathrm{~s}$. Also, a video showing behavior of the pupil during the measurement is recorded for documentation purposes. The software allows for working "with" and "without" calibrating the camera's optical system. Without calibration, it is possible to determine absolute or relative changes in 
pupil size. Absolute pupil sizes are measured in pixels (pix.), Fig. 4a. Relative values do not have a unit of measure, and they are in the $(0,1)$ range, Fig. $4 \mathrm{~b}$. In this case, the starting point for calculations is the highest measured pupil size. In such a configuration, "1" means the largest and " 0 " means the smallest pupil size.
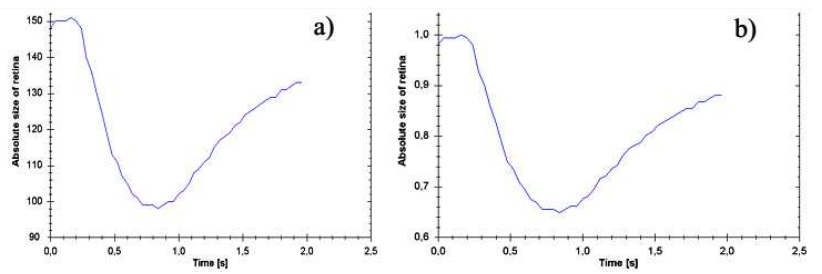

Fig. 4. Sample results of PLR measurements: absolute measurement in pix. (a) and relative (b).

Calibration allows for measuring the pupil size in millimeters. The procedure involves placing the object of known size in front of the sensor and pressing the "Calibration" button. The software will automatically search for an object similar to the shape of the pupil, measure and determine the number of millimeters per one pixel of the image. In order to maintain measurement accuracy during both calibration and normal operation, it is required that the contrast in the image was similar. For this purpose, the software implements a procedure for determining the contrast used in stills cameras (maximum contrast method), Fig. 5a.

a)

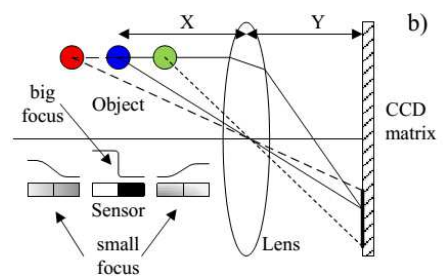

Fig. 5. Contrast measurement: principle of operation (a), location of the probes (b).

Contrast measurement is carried out using two probes placed on the left and right side of the pupil, Fig. 5b. Luminance values of pixels contained in the left and right part of the probe are summed for each image frame. Then the corresponding average luminance values $A$ and $B$ are determined. The result of the measurement suggests moving the camera so as to maximize the value of $|A-B|$. The optical system was calibrated using the template shown in Fig. 6a and b.

A circle with a diameter of $12 \mathrm{~mm}$ was selected from the template. After the calibration, the obtained measurement step for this diameter was $0.035398 \mathrm{~mm}$. Next, the software was switched to the "Measurement" mode, and a test measurement was made. The resulting value for the pattern was $12 \mathrm{~mm}$. For other elements of the template, the following values were obtained: $10 \mathrm{~mm}$ - a)

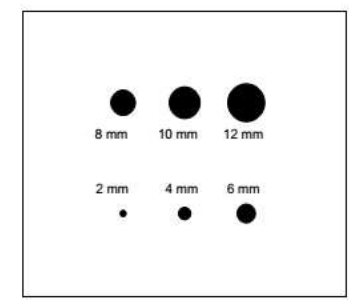

b)

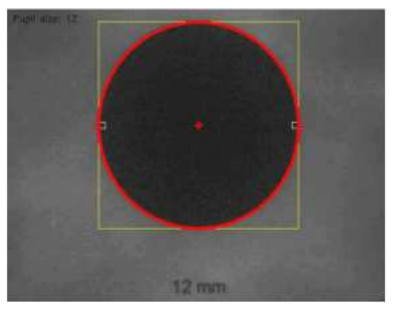

Fig. 6. Template (decreased) for the calibration of the measurement system (a) and measurement result (b).

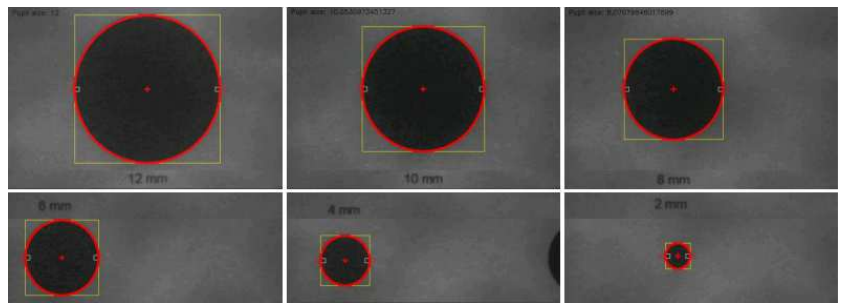

Fig. 7. Results of the test measurement of template elements.

$10.0531 \mathrm{~mm}, 8 \mathrm{~mm}-8.0708 \mathrm{~mm}, 6 \mathrm{~mm}-6.0177 \mathrm{~mm}$, $4 \mathrm{~mm}-4.0708 \mathrm{~mm}, 2 \mathrm{~mm}-2.0531 \mathrm{~mm}$, Fig. 7 .

As a result, the average measurement error for patterns was found to be $0.0442 \mathrm{~mm}$. This was followed by measuring changes in pupil size as a function of time after excitation with a red light pulse, Fig. 8. The results obtained show that the developed sensor can objectively assess pupil reaction to a stimulus in the form of a red light pulse. It also enables measurement of the variability range of pupil contraction, expressed in millimeters, as well as in relative and absolute manner.

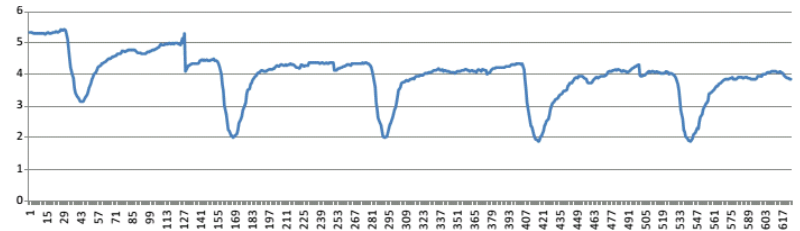

Fig. 8. Changes in pupil size as a function of time after the sequential $(5 \times)$ excitation with a red light pulse expressed in $\mathrm{mm}$.

Another important aspect was the safety of the sensor. Therefore, the IR illuminator of the sensor was tested and the results are shown in [17].

\section{Conclusions}

The tests provided an overview of the design requirements for the PLR optical sensor. They provided a guidance for the design of a system for detecting and identifying dynamic changes in the pupillary type reflex system. The designed proprietary optical sensor allowed for obtaining the required image acquisition speed 
(25 to $120 \mathrm{fps}$ ) and sufficient measurement accuracy $(0.0442 \mathrm{~mm})$. In practice, in the absence of a head-mounted housing, the only restriction on its use is the need for a darkroom. Along with the sensor, the authors have developed software capable of immediate visualization of the PLR reflex and video acquisition for documentation purposes.

The solution comes as part of a mobile pupillography device intended to be assembled in cars and airplanes to identify conditions of lower alertness, reduced ability to concentrate, increasing fatigue and drowsiness in drivers, pilots and traffic controllers, and to trigger alarm and preventive measures, if necessary [18].

\section{Acknowledgments}

The project was co-financed by the European Union through the European Regional Development Fund under the Innovative Economy Program. Development Project contract no. POIG.01.03.01-10-085/09.

\section{References}

[1] Ch. Tilmant, M. Charavel, M. Ponrouch, Monitoring and Modeling of Pupillary Dynamics: Study of the Autonomous Nervous System, Blaise Pascal University, OICMS France 2005.

[2] R.J. Duffey, D. Leaming, J. Refract. Surg. 21, 87 (2005).

[3] I. Loewenfeld, O. Lowenstein, Anatomy, Physiology, and Clinical Applications, Iowa State University Press, Ames 1993.

[4] A. Kawasaki, R.H. Kardon, Ophthalmol. Clin. North Am. 14, 149 (2001).
[5] D.F. Fotiou, V. Stergioua, D. Tsiptsiosa, C. Litharib, M. Nakouc, A. Karlovasitoua, Int. J. Psychophysiol. 73, 143 (2009).

[6] M. Hö̈le, R. Kenntner-Mabiala, P. Pauli, G.W. Alpers, J. Psychophysiol. 70, 171 (2008).

[7] J.W. McLaren, P.J. Hauri, Siong-Chi Lin, C. Harris, Sleep Medicine 3, 347 (2002).

[8] A. Kaltsatou, E. Kouidi, C. Lithari, N. Koutlianos, F. Fotiou, A. Deligiannis, Int. J. Psychophysiol. 77, 283 (2010).

[9] B. Wilhelm, A. Widmann, W. Durst, Ch. Heine, G. Otto, Int. J. Psychophysiol. 72, 307 (2009).

[10] M. Warga, H. Lüdtke, H. Wilhelm, B. Wilhelm, Vision Res. 49, 295 (2009).

[11] O. Lowenstein, R. Feinberg, I.E. Loewenfeld, Investigat. Ophthalmol. 2, 138 (1963).

[12] B. Wilhelm, H. Wilhelm, P. Streicher, H. Lüdtke, M. Adler, Wien Med. Wochenschr. 146, 387 (1996).

[13] K. Murawski, K. Różanowski, Acta Phys. Pol. A 124, 509 (2013).

[14] K. Gut, K. Nowak, Europ. Phys. J. Spec. Top. 154, 89 (2008).

[15] K. Murawski, in: Proc. XV Int. Conf. on Methods and Models in Automation and Robotics (MMAR), Międzyzdroje, Warsaw Univ., 2010, p. 356.

[16] K. Gut, Bull. Pol. Acad. Sci., Techn. Sci. 59, 395 (2011).

[17] M. Życzkowski, Acta Phys. Pol. A 122, 933 (2012).

[18] K. Murawski, Electrotechn. Rev. 9, 184 (2010). 